ARTICLE

Received 23 May 2013 | Accepted 19 Sep 2013 | Published 22 Oct 2013 DOI: 10.1038/ncomms3647

\title{
A solid-solid phase transition in carbon dioxide at high pressures and intermediate temperatures
}

Jinjin Li ${ }^{1}$, Olaseni Sode ${ }^{2}$, Gregory A. Voth ${ }^{2}$ \& So Hirata ${ }^{1,3}$

Despite its terrestrial abundance and astrochemical significance, many aspects of the phase diagram of solid carbon dioxide remain uncertain or unknown. The observed transition pressures from cubic to orthorhombic phase range widely from $2.5 \mathrm{GPa}$ at $80 \mathrm{~K}$ to above $18 \mathrm{GPa}$ at room temperature. The vibrational Raman bands that appear at higher pressure and serve as a decisive proof of the existence of the orthorhombic phase have never been assigned. Here we introduce a general ab initio computational method that can predict the Gibbs free energies and thus phase diagrams of molecular crystals. Using this with secondorder Møller-Plesset perturbation theory, we obtain the transition pressure of $13 \mathrm{GPa}$ at $0 \mathrm{~K}$ with small temperature dependence, which is in line with many experiments. We also computationally reproduce the vibrational Raman bands and explain the pressure dependence of the structure parameters and Raman band positions of both phases quantitatively.

\footnotetext{
${ }^{1}$ Department of Chemistry, University of Illinois at Urbana-Champaign, 600 South Mathews Avenue, Urbana, Illinois 61801, USA. ${ }^{2}$ Department of Chemistry, University of Chicago, 5735 South Ellis Avenue, Chicago, Illinois 60637, USA. ${ }^{3}$ CREST, Japan Science and Technology Agency, 4-1-8 Honcho, Kawaguchi, Saitama 332-0012, Japan. Correspondence and requests for materials should be addressed to S.H. (email: sohirata@illinois.edu).
} 
A $b$ initio computational prediction of crystal structures, spectra and phase diagrams has long been a goal of chemists, physicists and materials scientists. The difficulty of such prediction arises from the fact that atoms and molecules in a crystal are bound together by a variety of forces (covalent, ionic, hydrogen-bond and dispersion), whose strengths span three orders of magnitude ( 100 to $0.1 \mathrm{~kJ} \mathrm{~mol}^{-1}$ ). This may be compared with the free energy differences of a few $\mathrm{kJ} \mathrm{mol}^{-1}$ or less, determining the relative stability of crystalline polymorphs. Density functional theory (DFT), despite its usefulness and improvements in its approximations ${ }^{1}$, has fundamental limitations, two of the severest being the inability to systematically increase its accuracy and the lack of dispersion interactions. Systematic approximations of $a b$ initio molecular orbital theory must be extended to solids. The simplest in this category is the second-order Møller-Plesset perturbation (MP2) method, which can treat all of the aforementioned interactions accurately. However, the computational cost of MP2 is so great that its routine applications to solids are still difficult.

This situation is improving rapidly at least for a large and important class of solids known as molecular crystals. Molecular crystals are chemists' solids, so to speak, as their properties can be understood and even controlled by those of the constituent molecules. They include ices of atmospheric species ${ }^{2,3}$, drugs ${ }^{4,5}$ and explosives 6 . They are also related to organic superconductors and other functional materials ${ }^{7}$ as well as supramolecular assemblies ${ }^{8,9}$.

An example in the first category is solid carbon dioxide $\left(\mathrm{CO}_{2}\right)$, one of nature's most important molecular crystals. Despite its abundance in the Earth's atmosphere and ubiquity in other planets and asteroids in its ice forms, there remain many gaps in our knowledge of the phase diagram of $\mathrm{CO}_{2}^{2,10}$. Today, intense experimental efforts are devoted to explore the high pressure-temperature range in which non-molecular phases ${ }^{11-19}$ are observed. The phase diagram in the lower pressuretemperature range is relatively well characterized, yet some aspects of the phases there remain uncertain or unknown to this day.

More specifically, when Aoki et al. ${ }^{20}$ determined by x-ray diffraction the crystal structure of an orthorhombic (Cmca) phase, the dry ice or the cubic (Pa3) phase (I) had been the only established crystalline phase of $\mathrm{CO}_{2}$ (Fig. 1). The orthorhombic phase was identified as the high-pressure phase III initially detected by Hanson ${ }^{21}$ with Raman spectroscopy. This phase was also responsible for the slight volume collapse occurring at around $12 \mathrm{GPa}$ in the equation-of-state measurement of $\mathrm{Liu}^{22}$, who speculated a transition involving the tilting of the molecules. The reported transition pressures at room temperature range widely from $10 \mathrm{GPa}$ (ref. 20), $12 \mathrm{GPa}$ (ref. 23) to above $18 \mathrm{GPa}$ (ref. 21). The transition pressure upon unloading can be as low as a

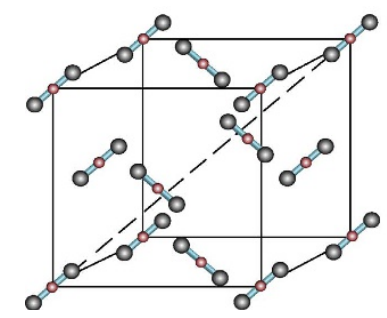

b Orthorhombic (Cmca)

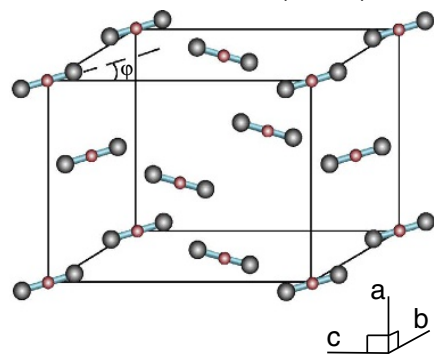

Figure 1 | Structures of $\mathbf{C O}_{\mathbf{2}}-\mathbf{I}$ and III. The $\mathrm{CO}_{2}$ molecules are in the bodydiagonal directions in (a) phase I ( $\mathrm{Pa} 3)$, whereas they are on the $b c$ planes in (b) phase III (Cmca). somewhere between 4.5 and $2.5 \mathrm{GPa}$ at $80 \mathrm{~K}$ (ref. 24). There is an extraordinary hysteresis in the transition, obscuring the precise transition pressure and its temperature dependence.

Interestingly, phase III is reached only by the low-temperature compression of phase $\mathrm{I}^{25}$. This implies that phase III is a metastable, kinetically trapped state; there is an underlying phase (II) ${ }^{17,26,27}$ with a lower free energy than phase III. Perhaps the most accurate phase diagram of $\mathrm{CO}_{2}$ available today is a composite obtained from the experimental data of Litasov et al. ${ }^{28}$, Giordano et al..$^{29,30}$ and Iota et al. ${ }^{17,25}$, which is reproduced in Fig. 2 (the broken line separating phases II and III is a kinetic line). This diagram seems consistent with the observation that, above $400 \mathrm{~K}$, the high-pressure phase transition occurs abruptly at $12-13 \mathrm{GPa}$, as opposed to sluggishly at lower temperatures.

The large hysteresis of the phase transition and the metastability of phase III make it difficult to characterize them by experimental means alone; accurate computational assistance is essential. In fact, the high-pressure phase transition from I to III was computationally predicted by Kuchta and Etters ${ }^{31-33}$ prior to the $\mathrm{x}$-ray diffraction study ${ }^{20}$. Their calculated value of the $\mathrm{CO}_{2}$ tilt angle $\varphi$ of $52^{\circ}$ was in exact agreement with the observed. Also, the DFT study by Bonev et al. ${ }^{34}$ ruled out the experimentally derived hypothesis ${ }^{12}$ that phase III was a high-strength material with the bulk modulus of $87 \mathrm{GPa}$ and was not a molecular crystal.

These pioneering calculations were, however, not without serious limitations. Those of Kuchta and Etters ${ }^{31-33}$ were based on empirical force fields and their predicted transition pressure of $4.3 \mathrm{GPa}$ was too low. The DFT calculations ${ }^{34}$ also lacked dispersion interactions, which were shown by us ${ }^{35}$ to be crucial cohesive forces in $\mathrm{CO}_{2}$-I. Furthermore, despite the fact that Hanson's Raman spectra ${ }^{21}$ were the first decisive proof of the existence of phase III, no direct simulation of the spectra supporting this assignment has yet been performed.

This article reports an advance in computational machinery ${ }^{36,37}$ that enables routine $a b$ initio calculations of the free energies of general molecular crystals at finite pressures ${ }^{38}$ and

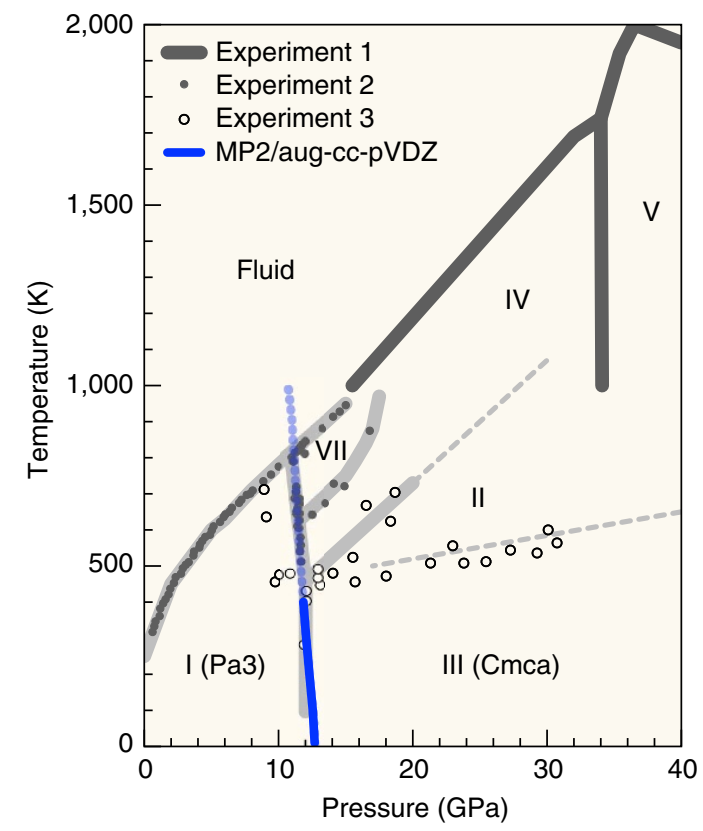

Figure 2 | Phase diagram. The calculated phase boundary between $\mathrm{CO}_{2}-\mid$ and III is superimposed on the experimentally inferred phase diagram of $\mathrm{CO}_{2}$ (data sets 1, 2 and 3 are from Litasov et al. ${ }^{28}$, Giordano et al. ${ }^{29,30}$ and lota et al. ${ }^{17,25}$, respectively). The boundary between phases II and III (thin broken line) is a kinetic line. 
temperatures and thus of phase diagrams from first principles. We demonstrate the utility of this method through its application to the structures, equations of state and Raman spectra of both phases I and III of $\mathrm{CO}_{2}$ as well as the solid-solid phase transition between them. We use MP2 with the exact electronic Hamiltonian (as opposed to empirical or modified Hamiltonians in previous calculations), which is the simplest theory that can describe all cohesive interactions in solid $\mathrm{CO}_{2}$ simultaneously, accurately and on an equal footing. MP2/aug-cc-pVDZ predicts the transition pressure of $13 \mathrm{GPa}$ (or a few $\mathrm{GPa}$ less at a larger basis set) at $0 \mathrm{~K}$ with small temperature dependence, which is in line with many experimental reports, while exposing the limitations of the DFT calculation ${ }^{34}$, predicting a higher transition pressure and greater temperature dependence, and of the empirical calculations ${ }^{31-33}$, yielding too low a transition pressure.

\section{Results}

Equations of state. The pressure-volume curves of $\mathrm{CO}_{2}$-I and III are drawn in Fig. 3. In Table 1, the calculated lattice constants and tilt angle $(\varphi)$ at $11.8 \mathrm{GPa}$ are compared with the experimental values ${ }^{20}$ and with those obtained with the empirical force field by Kuchta and Etters ${ }^{31}$. Note that in addition to $a, b, c$, and $\varphi$, the $\mathrm{CO}$ bond lengths in both $\mathrm{CO}_{2}$-I and III have also been optimized at each pressure in our calculations, which, therefore, constitute full optimization with only symmetry constraints imposed.

For both phases, MP2 with the aug-cc-pVDZ basis set predicts slightly more compact and stiffer solids at lower pressures than experiments ${ }^{20,39}$. This may be due to the overestimation of dispersion or quadrupole-quadrupole interactions or both in MP2. With increasing pressure, however, agreement between MP2 and experiments improves and becomes nearly exact (except for $c$ of phase III) at $11.8 \mathrm{GPa}$, where the $\mathrm{x}$-ray diffraction for $\mathrm{CO}_{2}$-III was performed ${ }^{20}$. This trend is commonly observed ${ }^{38}$. The accuracy of the empirical calculation of Kuchta and Etters ${ }^{31}$ reported before the $\mathrm{x}$-ray diffraction study is simply astonishing.

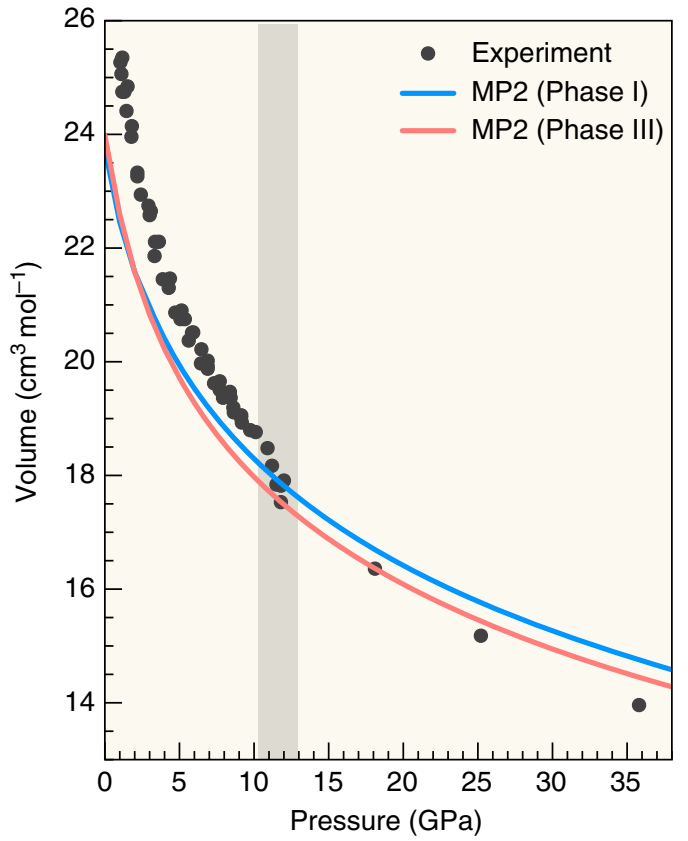

Figure 3 | Equations of state. The calculated pressure dependence of the volume of $\mathrm{CO}_{2}-\mathrm{I}$ and III is plotted along with the experimental data from $\mathrm{Liu}^{22}$. The latter seem to exhibit the volume collapse between 10-13 GPa (shaded area) as indication of a solid-solid phase transition.
Both our MP2 calculations and the one by Kuchta and Etters ${ }^{31}$ as well as the DFT study of Bonev et al. ${ }^{34}$ record $\sim 2 \%$ volume reduction upon transition from I to III. This volume difference persists (if not grows) with pressure according to both MP2 and DFT calculations. This is consistent with the experimental equation-of-state $\operatorname{plot}^{22}$ reproduced in Fig. 3, which shows a visible drop in the volume at about $12 \mathrm{GPa}$. Given the small temperature dependence of the transition pressure (see below), the driving force of this high-pressure transition may be this small volume reduction. The measured values of volume at pressures greater than $12 \mathrm{GPa}$ tend to fall on the MP2 results of phase III until MP2 again begins to predict slightly too stiff a solid. Overall, the structural data predicted by MP2 are fully consistent with the experimental data.

Figure 4 plots the $\mathrm{CO}$ bond lengths of both phases as a function of pressure. They decrease nearly linearly with pressure and by roughly $1 \%$ upon loading of $20 \mathrm{GPa}$. Despite the smaller volumes at most pressures, the molecules in phase III are more relaxed with systematically greater bond lengths than in phase I. This is most likely due to the preferable mutual orientations of $\mathrm{CO}_{2}$ in phase III than in phase I. The differences in $\mathrm{CO}$ bond length between the two phases also seem to grow with pressure, which may contribute at least partly to the relative stability of phase III

\begin{tabular}{|c|c|c|c|}
\hline Parameter ${ }^{\star}$ & Expt. $^{\dagger}$ & Calc..$^{\ddagger}$ & Calc. ${ }^{\S}$ \\
\hline$a(I) / \AA$ & $4.939 \pm 0.010$ & 4.92 & 4.91 \\
\hline$a(I I I) / \AA$ & $4.330 \pm 0.015$ & 4.17 & 4.33 \\
\hline$b(\mathrm{III}) / \AA$ & $4.657 \pm 0.005$ & 4.65 & 4.63 \\
\hline$c(I I I) / \AA$ & $5.963 \pm 0.009$ & 6.02 & 5.80 \\
\hline$\varphi($ III)/degree & 52 & 51.9 & 54.8 \\
\hline
\end{tabular}

${ }^{*} a$ (I) is the lattice constant of $\mathrm{CO}_{2}-\mathrm{I}(\mathrm{Pa} 3) . a(\mathrm{III}), b$ (III) and $c$ (III) are the lattice constants of $\mathrm{CO}_{2}$-III (Cmca). $\varphi$ (III) is the tilt angle (see Fig. 1) of $\mathrm{CO}_{2}-$ III.

†X-ray diffraction study at $11.8 \mathrm{GPa}$ (ref. 20).

Empirical-force-field calculations at $12 \mathrm{GPa}$ (ref. 31)

\$Second-order Møller-Plesset perturbation calculations at $11.8 \mathrm{GPa}$ (this work).

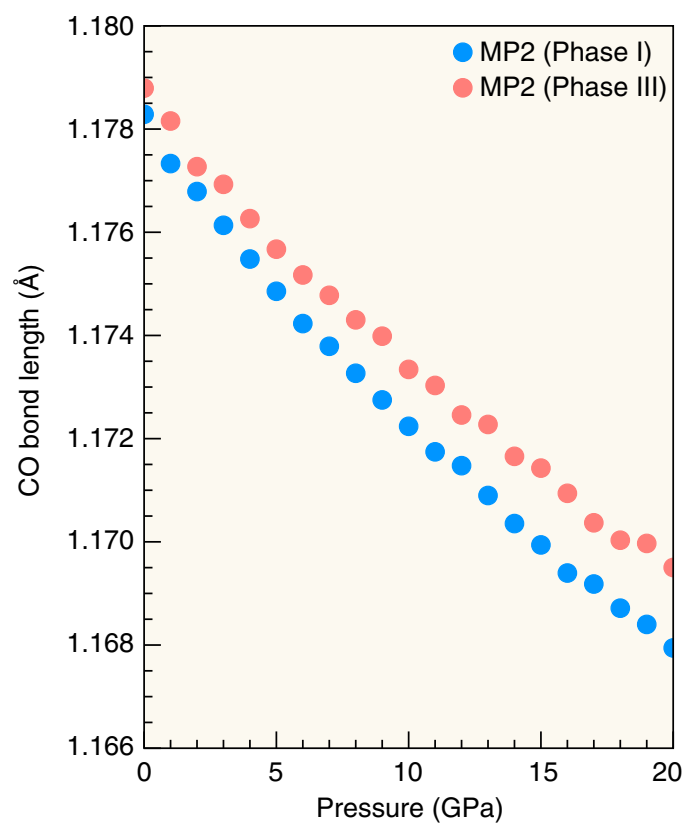

Figure 4 | $\mathbf{C O}$ bond lengths. The pressure dependence of the calculated $\mathrm{CO}$ bond lengths of $\mathrm{CO}_{2}-\mathrm{I}$ and III is plotted. 
over I at higher pressures. That the intramolecular structural and energy changes as well as intermolecular contributions are taken into account on an equal footing is one of the advantages of $a b$ initio methods.

Vibrational spectra. Next, we turn to the spectral data. Figure 5 compares the calculated and observed ${ }^{21}$ Raman spectra of $\mathrm{CO}_{2}-\mathrm{I}$ (at $14.5 \mathrm{GPa}$ ) and III (at 18.0 GPa). Upon pressure increase, three new Raman bands at 245, 270 and $320 \mathrm{~cm}^{-1}$ appear (top trace), serving as the most decisive proof of the existence of the new phase (III) suggested earlier by the equation-of-state measurement ${ }^{22}$. Our MP2 simulations reproduce the spectra of both phases with remarkable accuracy. $\mathrm{CO}_{2}-\mathrm{I}$ at $14.5 \mathrm{GPa}$ is predicted to display three intense Raman bands in the librational region, which are easily assigned to the three observed bands labelled $\mathrm{L}_{1}, \mathrm{~L}_{2}$ and $\mathrm{L}_{3}$ in Fig. 1 of Hanson ${ }^{21}$. $\mathrm{CO}_{2}$-III at $18.0 \mathrm{GPa}$ also has three discernible Raman bands in the simulated spectrum, but at rather different frequencies $\left(250-350 \mathrm{~cm}^{-1}\right)$, which are again readily assignable to the bands labelled 1, 2 and 3 by Hanson. The agreement in peak positions between theory and experiment is quantitative and the agreement in intensities is also satisfactory. This renders strong computational support for Hanson's assignment ${ }^{21}$ of the Raman bands at $18.0 \mathrm{GPa}$ to phase III.

Figure 6 shows the pressure dependence of these and other Raman band positions. While the observed Raman bands due to $\mathrm{CO}_{2}$-I (blue circles) are unmistakably identified because of the high symmetry of the crystal, it is not clear which additional observed features (red circles) belong to $\mathrm{CO}_{2}$-III above $12 \mathrm{GPa}$. Nevertheless, MP2 reproduces accurately the observed rates of frequency increase with pressure ${ }^{40}$.

Phase diagram. Given the accurate structures and phonon density of states (DOS) in the low frequency regions of $\mathrm{CO}_{2}$-I and III predicted by MP2, we are in a position to calculate the small salient portion of the phase diagram of $\mathrm{CO}_{2}$ and the transition

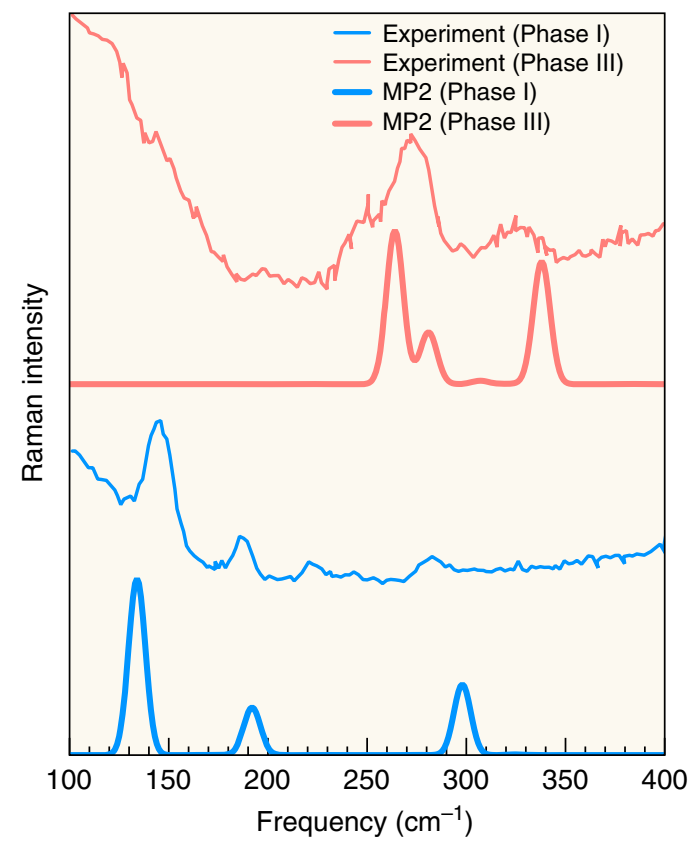

Figure 5 | Raman spectra. The calculated and observed ${ }^{21}$ Raman spectra of $\mathrm{CO}_{2}-\mathrm{I}$ (at $14.5 \mathrm{GPa}$ ) and III (at $18.0 \mathrm{GPa}$ ) are compared in the librational region. The calculated spectra are convoluted with a Gaussian with a fullwidth half-maximum of $10 \mathrm{~cm}^{-1}$. pressure as a function of temperature. Our MP2 result of the phase boundary between I and III is superimposed as a blue curve in Fig. 2. MP2 predicts the transition pressure to be $12.7 \mathrm{GPa}$ at $10 \mathrm{~K}$, which slightly decreases to $12.6 \mathrm{GPa}$ at $100 \mathrm{~K}, 12.3 \mathrm{GPa}$ at $200 \mathrm{~K}, 12.1 \mathrm{GPa}$ at $300 \mathrm{~K}$ and $11.9 \mathrm{GPa}$ at $400 \mathrm{~K}$. The calculated boundary above this temperature (blue broken line) is probably not meaningful, given the other phases experimentally inferred to exist there.

These calculated transition pressures are slightly above the observed range of 10-12 GPa (see the introductory paragraphs). The small temperature dependence, that is, the nearly vertical phase boundary between $\mathrm{CO}_{2}$-I and III, is also consistent with the small range of median transition pressures measured at various temperatures between $40 \mathrm{~K}$ to room temperature. The DFT calculations by Bonev et al. ${ }^{34}$ predict the transition pressures that depend strongly on temperature, going from about $16 \mathrm{GPa}$ at $0 \mathrm{~K}$ to about $11 \mathrm{GPa}$ at $350 \mathrm{~K}$, which disagrees with our MP2 results. The experimental data, although not clearcut because of the large hysteresis, seems more in line with MP2 than DFT. Interestingly, DFT predicts $^{34}$ a vertical phase boundary at $11 \mathrm{GPa}$ between $\mathrm{CO}_{2}$-I and II $\left(\mathrm{P}_{2} / \mathrm{mmm}\right)$. This result should not be taken to indicate that what was believed to be $\mathrm{CO}_{2}$-III (Cmca) in many experiments was indeed $\mathrm{CO}_{2}$-II $\left(\mathrm{P}_{2} / \mathrm{mmm}\right)$; our $\mathrm{MP} 2$ calculations on the structure, spectra and phase boundary are all consistent with the interpretation that phase III is Cmca.

Gibbs free energies. Figure 7 shows the free energy differences between phases I and III as a function of pressure and temperature. The general shape of the curves predicted by MP2 is similar to the one from $\mathrm{DFT}^{34}$, including the maximum at a few GPa except that the transition pressure predicted by DFT seems too high. With increasing pressure, the effect of temperature diminishes and the five MP2 curves at five different temperatures converge toward one. This is expected and traced to the hardening of acoustic phonons at high pressures. The free energy difference predicted by the empirical force field ${ }^{31}$ differs

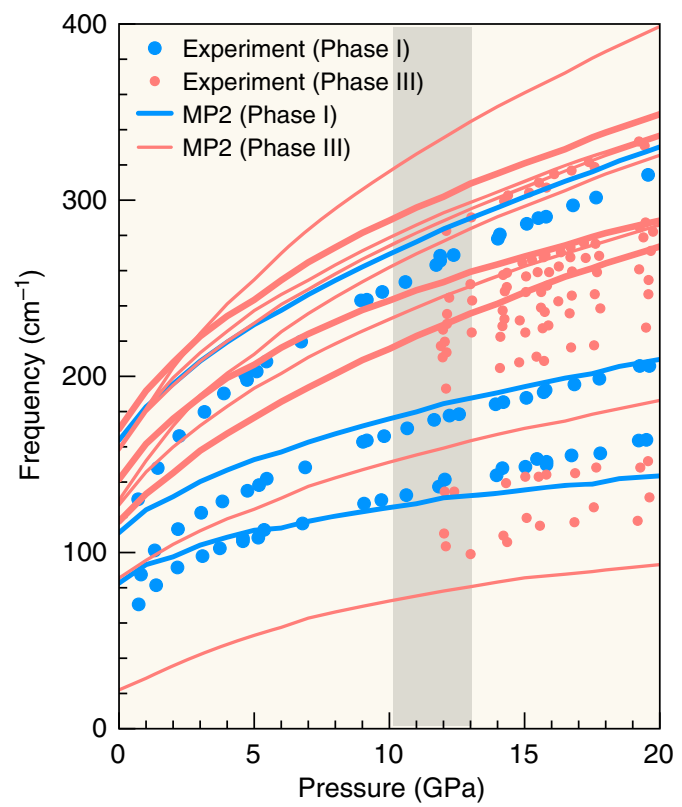

Figure 6 | Raman band positions. The pressure dependence of the calculated and observed ${ }^{40}$ frequencies of the Raman bands of $\mathrm{CO}_{2}-\mathrm{I}$ and III is plotted in the librational region. The bold curves in red correspond to the three prominent Raman peaks of $\mathrm{CO}_{2}$-III in Fig. 5. 


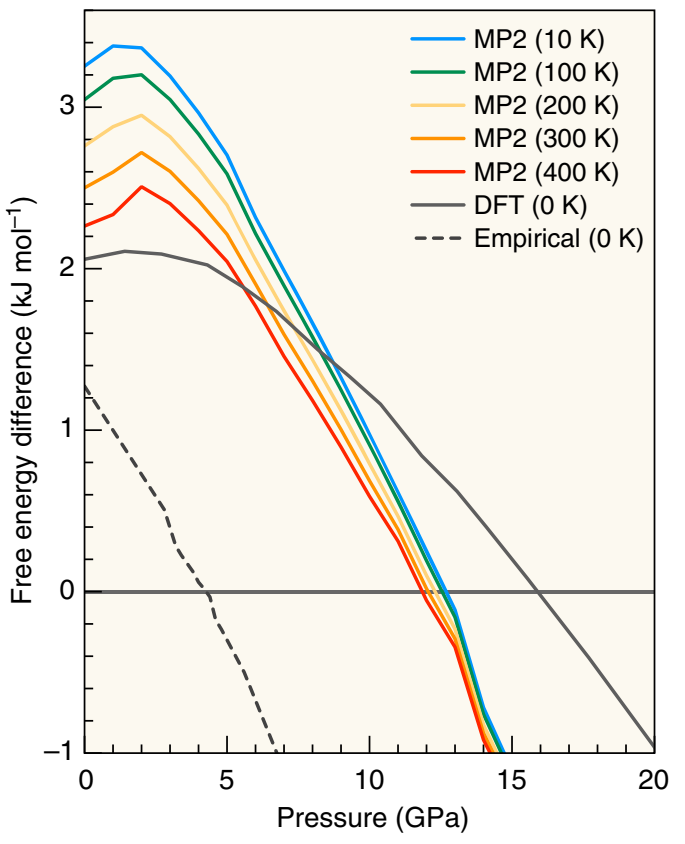

Figure 7 | Gibbs free energies. The Gibbs free energy differences between $\mathrm{CO}_{2} \mathrm{I}$ and III (a positive value means that phase I is more stable) are given as a function of pressure. The solid curves are from second-order MøllerPlesset perturbation calculations (this work) except for the grey curve, which is from the density functional calculations of Bonev et al. ${ }^{34}$ The broken curve is from the empirical-force-field calculations of Kuchta and Etters $^{31}$.

greatly from MP2 or DFT and is likely wrong. Given the success of the empirical force field in predicting the crystal structures, this implies the importance of intramolecular structural changes upon compression (see Fig. 4), an effect included in MP2 and DFT, but not in the empirical method.

The largest source of errors in our calculations is most likely the basis set ${ }^{41}$ and its impact is the greatest in the free energy differences. We have, therefore, performed MP2/aug-cc-pVTZ calculations on enthalpy differences between the two phases. The results obtained with the aug-cc-pVTZ basis set are found to be $+2.2,-2.1$ and $-3.5 \mathrm{~kJ} \mathrm{~mol}^{-1}$ at 0,14 and $18 \mathrm{GPa}$ (a positive value means that phase I is more stable), which are approximately $1.5 \mathrm{~kJ} \mathrm{~mol}^{-1}$ lower than the corresponding values $(+3.6,-0.5$ and $-2.0 \mathrm{~kJ} \mathrm{~mol}^{-1}$, respectively) obtained with the aug-ccpVDZ basis set. These suggest that the free energy difference curves shown in Fig. 7 are qualitatively correct, but a basis-set extension stabilizes phase III more than phase I and shifts the IIII transition pressure to a lower value by a few GPa, possibly for better agreement with experiments. Other potentially significant sources of errors are discussed in the Methods section and include higher-order correlation and three-body dispersion effects ${ }^{42,43}$. All must be taken into account to reach the so-called 'chemical accuracy $^{44}$ of $4 \mathrm{~kJ} \mathrm{~mol}^{-1}$ in energy differences.

\section{Discussion}

We have developed an $a b$ initio electron-correlated method generally applicable to periodic and nonperiodic molecular crystals at finite pressures and temperatures, which, therefore, reports Gibbs free energies and phase diagrams from first principles. It is based on an embedded-fragment approach and can be combined with any electron-correlation theory including but not limited to MP2. Unlike planewave-based methods for crystals, this method can compute phonon dispersion in the entire reciprocal space without a costly supercell approach in addition to the frequencies and intensities of the infrared and Raman-active phonons occurring at the centre of the reciprocal unit cell. It can, therefore, calculate phonon DOS and finitetemperature effects.

With this method at the MP2/aug-cc-pVDZ level, we have reproduced quantitatively the frequencies and intensities of the librational Raman bands ${ }^{21}$ of both phases I and III using their respective structures, force constants and polarizability derivatives. This supports the original assignments of Hanson ${ }^{21}$. MP2/aug-cc-pVDZ places the transition pressure from phase I to $\mathrm{III}$ at $13 \mathrm{GPa}$ at $0 \mathrm{~K}$ with small temperature dependence, whereas the basis-set extension to aug-cc-pVTZ lowers the transition pressures by a few GPa, possibly for better agreement with experiments. These are in contrast with the previous DFT study $^{34}$, predicting the transition pressure of $16 \mathrm{GPa}$ at $0 \mathrm{~K}$ with much greater temperature dependence. MP2 does not agree with the empirical calculations ${ }^{31}$, the latter proposing a very low transition pressure of $4.3 \mathrm{GPa}$. The existing body of experimental evidence is more in line with the MP2 results.

The $a b$ initio computational method reported here, which can be parallel executed routinely on small computer clusters for infinite three-dimensional solids, therefore, holds exceptional promise in assisting experimental studies of molecular crystals, which are important in high-pressure chemistry ${ }^{2,3,6}$, solid-state physics $^{3,7}$, geochemistry ${ }^{2}$, astrophysics ${ }^{3}$, medicinal chemistry ${ }^{4,5}$, supramolecular chemistry ${ }^{8,9}$, materials science ${ }^{4,7}$ and more.

\section{Methods}

Internal energy. Our MP2 calculation of the Gibbs free energy of a molecular crystal is based on an embedded-fragment scheme $e^{36-38}$, which, in turn, uses the Bethe-Goldstone expansion of the total energy ${ }^{45,46}$.

The internal energy per unit cell, $U_{\mathrm{e}}$, of solid $\mathrm{CO}_{2}$ is approximated by

$$
U_{\mathrm{e}}=\sum_{i} E_{i(0)}+\frac{1}{2} \sum_{\mathbf{n}} \sum_{i, j}^{\prime}\left\{E_{i(\mathbf{0}) j(\mathbf{n})}-E_{i(\mathbf{0})}-E_{j(\mathbf{n})}\right\}+E_{\mathrm{LR}},
$$

where $\mathbf{n}$ is a three-integer index of a unit cell, $E_{i(\mathbf{n})}$ is, in our case, the MP2/aug-ccpVDZ energy of the $i$ th $\mathrm{CO}_{2}$ molecule in the $\mathbf{n}$ th unit cell, $E_{i(\mathbf{0}) j(\mathbf{n})}$ is the MP2/augcc-pVDZ energy of the dimer consisting of the $i$ th molecule in the 0 th unit cell and the $j$ th molecule in the $\mathbf{n}$ th unit cell. The prime on the summation symbol indicates that the spurious $E_{i(\mathbf{0}) i(\mathbf{0})}$ contributions are to be left out of the summation. In this work, the summation over $\mathbf{n}$ has been taken in the $3 \times 3 \times 3$ supercell, enclosing 422 dimers.

These monomers and dimers are placed in the electrostatic field of the rest of the crystal represented by atomic charges computed by the method developed by Kamiya et al. ${ }^{47}$ at the Hartree-Fock (HF) level with the same basis set. These atomic charges are furthermore brought to self consistency with one another ${ }^{36}$. We have taken into account the atomic charges within the $11 \times 11 \times 11$ supercell, consisting of 5,324 molecules.

The last term in equation (1), $E_{\mathrm{LR}}$, is the long-range electrostatic contributions and a part of the Madelung constant ${ }^{37,48}$. It has been computed with the same atomic charges within the supercell of dimension $41 \times 41 \times 41$, having over a quarter million molecules. Hence, our calculations take into account the 422 most important pairwise electrostatic (that is, induction and hydrogen-bond cooperativity), exchange and correlation (that is, dispersion) interactions at the MP2 level as well as three-body and all higher-order many-body electrostatic interactions within $\sim 20 \AA$ at the HF level. The additional long-range pairwise electrostatic interactions have also been included up to nearly $100 \AA$ or even farther.

Enthalpy. The effect of pressure has been incorporated ${ }^{38}$ by calculating the enthalpy per unit cell,

$$
H_{\mathrm{e}}=U_{\mathrm{e}}+P V,
$$

where $P$ is the pressure and $V$ is the unit cell volume, that is, $a^{3}$ (phase I) or $a b c$ (phase III). Both $H_{\mathrm{e}}$ and $U_{\mathrm{e}}$ can be analytically differentiated with atomic positions and lattice constants ${ }^{37,38}$. With these, we have determined the equilibrium

structures and phonon dispersion and DOS of $\mathrm{CO}_{2}$-I and III under pressure up to 20.0 GPa. The pressure due to phonons is neglected. We have repeated the singlepoint enthalpy calculations at the MP2/aug-cc-pVTZ level at 0,14 and $18 \mathrm{GPa}$ at their respective MP2/aug-cc-pVDZ optimized geometries to assess the effect of a basis-set extension on phase I-III enthalpy differences. Additionally, we have 
calculated the Raman frequencies and intensities of the zone-centre phonons of $\mathrm{CO}_{2}$-I at $14.5 \mathrm{GPa}$ and $\mathrm{CO}_{2}$-III at $18.0 \mathrm{GPa}$ using the method developed by He et al. ${ }^{49}$

Gibbs free energy. The Gibbs free energy per unit cell, $G$, at temperature $T$ is obtained by evaluating

$$
G=H_{\mathrm{e}}+U_{\mathrm{v}}-T S_{\mathrm{v}},
$$

where $U_{\mathrm{v}}$ is the zero-point vibrational energy per unit cell at $T$ and $S_{\mathrm{v}}$ is the vibrational entropy per unit cell. For a molecular crystal, which has a large band gap, only the temperature effects due to phonons need to be considered. Both $U_{\mathrm{v}}$ and $S_{\mathrm{v}}$ are derivable from the partition function of phonons, $Z_{\mathrm{v}}$. In the harmonic approximation and atomic units, it is written as ${ }^{50,51}$

$$
Z_{\mathrm{v}}=\prod_{n} \prod_{\mathbf{k}} \frac{e^{-\beta \omega_{n \mathrm{k}} / 2}}{1-e^{-\beta \omega_{n \mathrm{k}}}},
$$

where $\beta=\left(k_{\mathrm{B}} T\right)^{-1}, k_{\mathrm{B}}$ is the Boltzmann constant, and $\omega_{n \mathbf{k}}$ is the frequency of the phonon in the $n$th phonon branch with the wave vector $\mathbf{k}$. The latter is determined at the equilibrium structure where $H_{\mathrm{e}}($ not $G$ ) is a minimum. The product over $\mathbf{k}$ must be taken over all $K$ evenly spaced grid points of $\mathbf{k}$ in the reciprocal unit cell. In this study, the k-grid of $21 \times 21 \times 21$ has been used $(K=9,261)$. According to thermodynamics ${ }^{50,51}$, we have

$$
\begin{gathered}
U_{\mathbf{v}}=\frac{T}{\beta K} \frac{\partial \ln Z_{\mathrm{v}}}{\partial T} \\
=\frac{1}{K} \sum_{n} \sum_{\mathbf{k}} \omega_{n \mathbf{k}}\left(\frac{1}{2}+\frac{1}{e^{\beta \omega_{n \mathbf{k}}}-1}\right), \\
S_{\mathrm{v}}=\frac{1}{\beta K} \frac{\partial \ln Z_{\mathrm{v}}}{\partial T}+\frac{1}{\beta T K} \ln Z_{\mathrm{v}} \\
=\frac{1}{\beta T K} \sum_{n} \sum_{\mathbf{k}}\left\{\frac{\beta \omega_{n \mathbf{k}}}{e^{\beta \omega_{n \mathbf{k}}}-1}-\ln \left(1-e^{-\beta \omega_{n \mathbf{k}}}\right)\right\} .
\end{gathered}
$$

The right-hand sides of these equations can be readily evaluated with phonon dispersion, $\left\{\omega_{n \mathbf{k}}\right\}$. The availability of the latter in the entire reciprocal space (not just at the $\Gamma$ point) is one of the unique advantages of the fragment schemes unconstrained by the periodic boundary conditions.

Computer implementation. The calculations have been performed by our inhouse parallel execution programme, running monomer and dimer MP2 calculations using unmodified Gaussian0952. The structural optimization, vibrational analyses and free energy calculations have been performed with our in-house software.

Known limitations. Although this constitutes a state-of-the-art calculation for solids and a significant improvement over DFT or empirical calculations, it has numerous sources of errors. The gravest is the approximate nature of MP2 and the incompleteness of the basis sets ${ }^{41,44}$. The use of the harmonic approximation in the treatment of phonons is responsible for errors at higher temperatures ${ }^{49}$. Another source of errors is the use of HF in determining the embedding field and its truncation at a finite distance. It also neglects three-body and higher many-body exchange and correlation interactions, which have been shown ${ }^{42,43}$ to be nonnegligible for $\mathrm{CO}_{2}$. The agreement between experiment and simulation in this study, therefore, implicitly exploits the cancellation of these errors between two similar structures or phases. However, it should be emphasized that, thanks to the systematic nature of the $a b$ initio molecular orbital theory and embedded-fragment scheme, all of these errors can, in principle, be arbitrarily reduced at an increased computational cost.

\section{References}

1. Burke, K. Perspective on density functional theory. J. Chem. Phys. 136, 150901 (2012).

2. Hemley, R. J. Effects of high pressure on molecules. Annu. Rev. Phys. Chem. 51, 763-800 (2000).

3. McMahon, J. M., Morales, M. A., Pierleoni, C. \& Ceperley, D. M. The properties of hydrogen and helium under extreme conditions. Rev. Mod. Phys. 84, 1607-1653 (2012).

4. Bernstein, J. Polymorphism in Molecular Crystals (Clarendon Press, 2002).

5. Wen, S. H. \& Beran, G. J. O. Accidental degeneracy in crystalline aspirin: new insights from high-level ab initio calculations. Cryst. Growth Des. 12, 2169-2172 (2012).

6. Dlott, D. D. Ultrafast spectroscopy of shock waves in molecular materials. Annu. Rev. Phys. Chem. 50, 251-278 (1999).

7. Ishiguro, T., Yamaji, K. \& Saito, G. Organic Superconductors (Springer-Verlag, 1998).
8. Lieserowitz, L. \& Stoddart, J. F. Molecular crystals. Curr. Opin. Solid St. Mat. Sci. 3, 397-398 (1998).

9. Inokuma, Y., Kawano, M. \& Fujita, M. Crystalline molecular flasks. Nature Chem. 3, 349-358 (2011).

10. Santoro, M. \& Gorelli, F. A. High pressure solid state chemistry of carbon dioxide. Chem. Soc. Rev. 35, 918-931 (2006).

11. Iota, V., Yoo, C. S. \& Cynn, H. Quartzlike carbon dioxide: an optically nonlinear extended solid at high pressures and temperatures. Science $\mathbf{2 8 3}$, 1510-1513 (1999).

12. Yoo, C. S. et al. Crystal structure of carbon dioxide at high pressure: 'superhard' polymeric carbon dioxide. Phys. Rev. Lett. 83, 5527-5530 (1999).

13. Yoo, C. S., Iota, V. \& Cynn, H. Nonlinear carbon dioxide at high pressures and temperatures. Phys. Rev. Lett. 86, 444-447 (2001).

14. Tschauner, O., Mao, H. K. \& Hemley, R. J. New transformations of $\mathrm{CO}_{2}$ at high pressures and temperatures. Phys. Rev. Lett. 87, 075701 (2001).

15. Santoro, M., Lin, J.-F., Mao, H.-K. \& Hemley, R. J. In situ high P-T Raman spectroscopy and laser heating of carbon dioxide. J. Chem. Phys. 121, 2780-2787 (2004).

16. Santoro, M. et al. Amorphous silica-like carbon dioxide. Nature 441, 857-860 (2006).

17. Iota, V. et al. Six-fold coordinated carbon dioxide VI. Nature Mater. 6, 34-38 (2007).

18. Montoya, J. A., Rousseau, R., Santoro, M., Gorelli, F. \& Scandolo, S. Mixed threefold and fourfold carbon coordination in compressed $\mathrm{CO}_{2}$. Phys. Rev. Lett. 100, 163002 (2008)

19. Yoo, C. S., Sengupta, A. \& Kim, M. Phase diagram of carbon dioxide: update and challenges. High Press. Res. 31, 68-74 (2011).

20. Aoki, K., Yamawaki, H., Sakashita, M., Gotoh, Y. \& Takemura, K. Crystal structure of the high-pressure phase of solid $\mathrm{CO}_{2}$. Science 263, 356-358 (1994).

21. Hanson, R. C. A new high-pressure phase of solid $\mathrm{CO}_{2}$. J. Phys. Chem. 89, 4499-4501 (1985).

22. Liu, L. G. Compression and phase-behavior of solid $\mathrm{CO}_{2}$ to half a megabar. Earth Planet. Sci. Lett. 71, 104-110 (1984).

23. Aoki, K., Yamawaki, H. \& Sakashita, M. Phase study of solid $\mathrm{CO}_{2}$ to $20 \mathrm{GPa}$ by infrared-absorption spectroscopy. Phys. Rev. B 48, 9231-9234 (1993).

24. Olijnyk, H., Daufer, H., Jodl, H. J. \& Hochheimer, H. D. Effect of pressure and temperature on the Raman spectra of solid $\mathrm{CO}_{2}$. J. Chem. Phys. 88, 4204-4212 (1988).

25. Iota, V. \& Yoo, C. S. Phase diagram of carbon dioxide: evidence for a new associated phase. Phys. Rev. Lett. 86, 5922-5925 (2001).

26. Liu, L. G. Dry ice II, a new polymorph of $\mathrm{CO}_{2}$. Nature 303, 508-509 (1983).

27. Yoo, C. S. et al. Crystal structure of pseudo-six-fold carbon dioxide phase II at high pressures and temperatures. Phys. Rev. B 65, 104103 (2002).

28. Litasov, K. D., Goncharov, A. F. \& Hemley, R. J. Crossover from melting to dissociation of $\mathrm{CO}_{2}$ under pressure: implications for the lower mantle. Earth Planet. Sci. Lett. 309, 318-323 (2011)

29. Giordano, V. M., Datchi, F. \& Dewaele, A. Melting curve and fluid equation of state of carbon dioxide at high pressure and high temperature. J. Chem. Phys. 125, 054504 (2006).

30. Giordano, V. M. \& Datchi, F. Molecular carbon dioxide at high pressure and high temperature. Europhys. Lett. 77, 46002 (2007).

31. Kuchta, B. \& Etters, R. D. Prediction of a high-pressure phase transition and other properties of solid $\mathrm{CO}_{2}$ at low-temperatures. Phys. Rev. B 38, 6265-6269 (1988).

32. Etters, R. D. \& Kuchta, B. Static and dynamic properties of solid $\mathrm{CO}_{2}$ at various temperatures and pressures. J. Chem. Phys. 90, 4537-4541 (1989).

33. Kuchta, B. \& Etters, R. D. Generalized free-energy method used to calculate the high-pressure, high-temperature phase transition in solid $\mathrm{CO}_{2}$. Phys. Rev. B 47, 14691-14695 (1993).

34. Bonev, S. A., Gygi, F., Ogitsu, T. \& Galli, G. High-pressure molecular phases of solid carbon dioxide. Phys. Rev. Lett. 91, 065501 (2003).

35. Sode, O., Keceli, M., Yagi, K. \& Hirata, S. Fermi resonance in solid $\mathrm{CO}_{2}$ under pressure. J. Chem. Phys. 138, 074501 (2013).

36. Hirata, S. et al. Fast electron correlation methods for molecular clusters in the ground and excited states. Mol. Phys. 103, 2255-2265 (2005).

37. Hirata, S. Fast electron-correlation methods for molecular crystals: an application to the $\alpha, \beta_{1}$, and $\beta_{2}$ polymorphs of solid formic acid. J. Chem. Phys. 129, 204104 (2008).

38. Sode, O. \& Hirata, S. Second-order many-body perturbation study of solid hydrogen fluoride under pressure. Phys. Chem. Chem. Phys. 14, 7765-7779 (2012).

39. Olinger, B. The compression of solid $\mathrm{CO}_{2}$ at $296 \mathrm{~K}$ to $10 \mathrm{GPa}$. J. Chem. Phys. 77, 6255-6258 (1982).

40. Olijnyk, H. \& Jephcoat, A. P. Vibrational studies on $\mathrm{CO}_{2}$ up to $40 \mathrm{GPa}$ by Raman spectroscopy at room temperature. Phys. Rev. B 57, 879-888 (1998).

41. Beran, G. J. O. \& Nanda, K. Predicting organic crystal lattice energies with chemical accuracy. J. Phys. Chem. Lett. 1, 3480-3487 (2010). 
42. Persson, R. A. X. Gaussian charge polarizable interaction potential for carbon dioxide. J. Chem. Phys. 134, 034312 (2011).

43. Yu, K. \& Schmidt, J. R. Many-body effects are essential in a physically motivated $\mathrm{CO}_{2}$ force field. J. Chem. Phys. 136, 034503 (2012).

44. Helgaker, T., Klopper, W. \& Tew, D. P. Quantitative quantum chemistry. Mol. Phys. 106, 2107-2143 (2008).

45. Gordon, M. S., Fedorov, D. G., Pruitt, S. R. \& Slipchenko, L. V. Fragmentation methods: a route to accurate calculations on large systems. Chem. Rev. 112, 632-672 (2012).

46. Beran, G. J. O. \& Hirata, S. Fragment and localized orbital methods in electronic structure theory. Phys. Chem. Chem. Phys. 14, 7559-7561 (2012).

47. Kamiya, M., Hirata, S. \& Valiev, M. Fast electron correlation methods for molecular clusters without basis set superposition errors. J. Chem. Phys. 128, 074103 (2008).

48. Sode, O. \& Hirata, S. Second-order many-body perturbation study of solid hydrogen fluoride. J. Phys. Chem. A 114, 8873-8877 (2010).

49. He, X., Sode, O., Xantheas, S. S. \& Hirata, S. Second-order many-body perturbation study of ice Ih. J. Chem. Phys. 137, 204505 (2012).

50. McQuarrie, D. A. \& Simon, J. D. Molecular Thermodynamics (University Science Books, 1999).

51. Wallace, D. C. Thermodynamics of Crystals (Dover, 1972).

52. Frisch, M. J., Trucks, G. W. \& Schlegel, H. B. et al. Gaussian 09. Revision B.01 (Gaussian Inc., 2010).

\section{Acknowledgements}

J.L. and S.H. thank the financial support provided by the Scientific Discovery through Advanced Computing (SciDAC) programme funded by U.S. Department of Energy, Office of Science, Basic Energy Sciences under grant no. DE-FG02-12ER46875. O.S. was partially supported by the National Science Foundation under grant no. CHE-1118616. G.A.V. was supported by the National Science Foundation under grant no. CHE1214087. S.H. is a Camille Dreyfus Teacher-Scholar and a Scialog Fellow of the Research Corporation for Science Advancement. Professor Xiao He is thanked for useful advice.

\section{Author contributions}

The research was designed by O.S. and S.H., the computer programme was written by J.L. and O.S., and the calculation was performed by J.L. The results were analysed by all. The paper was written by J.L. and revised by S.H. and G.A.V.

\section{Additional information}

Competing financial interests: The authors declare no competing financial interests.

Reprints and permission information is available online at http://npg.nature.com/ reprintsandpermissions/

How to cite this article: Li, J. et al. A solid-solid phase transition in carbon dioxide at high pressures and intermediate temperatures. Nat. Commun. 4:2647 doi: 10.1038/ncomms3647 (2013) 


\section{Corrigendum: A solid-solid phase transition in carbon dioxide at high pressures and intermediate temperatures}

Jinjin Li, Olaseni Sode, Gregory A. Voth \& So Hirata

Nature Communications 4:2647 doi: 10.1038/ncomms3647 (2013); Published 22 Oct 2013; Updated 6 Nov 2015

In Table 1 of this article, the values of lattice constants $a$ and $b$ of $\mathrm{CO}_{2}$-III calculated by second-order Møller-Plesset perturbation theory ('4.33' and ' 4.63 ' in the rightmost column) were inadvertently swapped. The correct version of Table 1 appears below.

\begin{tabular}{|c|c|c|c|}
\hline Parameter $^{\star}$ & Expt. $^{\dagger}$ & Calc. ${ }^{\ddagger}$ & Calc. ${ }^{\S}$ \\
\hline$a(1) / \AA$ & $4.939 \pm 0.010$ & 4.92 & 4.91 \\
\hline$a(I I I) / \AA$ & $4.330 \pm 0.015$ & 4.17 & 4.63 \\
\hline$b(I I I) / \AA$ & $4.657 \pm 0.005$ & 4.65 & 4.33 \\
\hline$c(I I I) / \AA$ & $5.963 \pm 0.009$ & 6.02 & 5.80 \\
\hline$\varphi(I I I) /$ degree & 52 & 51.9 & 54.8 \\
\hline
\end{tabular}

